\title{
The Coordination of T-cell Function by Serine/Threonine Kinases
}

\author{
David Finlay and Doreen Cantrell \\ Division of Cell Biology and Immunology, University of Dundee, Dundee, United Kingdom \\ Correspondence: d.a.cantrell@dundee.ac.uk
}

The function of T-lymphocytes during adaptive immune responses is directed by antigen receptors, costimulatory molecules, and cytokines. These extrinsic stimuli are coupled to a network of serine/threonine kinases that control the epigenetic, transcriptional, and metabolic programs that determine T-cell function. It is increasingly recognized that serine/threonine kinases, notably those that are controlled by lipid second messengers such as polyunsaturated diacylglycerols (DAG) and phosphatidylinositol-(3,4,5)-trisphosphate $\left(\mathrm{PIP}_{3}\right)$, are at the core of T-cell signal transduction. In the present review the object will be to discuss some important examples of how pathways of serine/threonine phosphorylation control molecular functions of proteins and control protein localization to coordinate T-cell function in adaptive immune responses.

$T^{\mathrm{h}}$ he magnitude and complexity of the Tcell serine/threonine phospho-proteome is beginning to be appreciated. In this context, major technological advances in mass spectrometry have allowed large-scale quantitative phospho-proteomic analyses of T-cells. For example, recent studies have identified approximately 10,000 phosphorylations in the T-leukemic cell line Jurkat of which more than 600 were regulated in response to triggering of the T-cell antigen receptor (Carrascal et al. 2008; Mayya et al. 2009; Nguyen et al. 2009). The complexity and diversity of this network of protein phosphorylation in activated $\mathrm{T}$ cells is striking and raises many questions not least what is the role of the individual protein phosphorylations for T-cell biology. A simplistic view of protein phosphorylation is that it can act as an on/off switch to control protein structure/function; the other key impact of protein phosphorylation is to promote protein interactions that can modify protein activity and subcellular localization. The other important consideration is that kinases are enzymes and function to amplify signal transduction pathways. The present review will not exhaustively list the pathways of serine/threonine phosphorylation in $T$ cells but rather discuss core elements and key issues about the network of serine/ threonine kinases that control T-cell function.

\section{PROTEIN KINASES AS ON/OFF SWITCHES IN T CELLS}

At a basic level serine/threonine kinases can work as a series of on/off switches that are tightly coupled to triggering of antigen and cytokine

Editors: Lawrence E. Samelson and Andrey S. Shaw

Additional Perspectives on Immunoreceptor Signaling available at www.cshperspectives.org

Copyright (C) 2011 Cold Spring Harbor Laboratory Press; all rights reserved; doi: 10.1101/cshperspect-a002261

Cite this article as Cold Spring Harb Perspect Biol 2011;3:a002261 
D. Finlay and D. Cantrell

receptors. There are numerous examples of kinases activated by protein phosphorylation that fit this concept in T-cells (Fig. 1). For example, T-cell antigen receptor (TCR) triggering stimulates phospholipase C (PLC)-mediated hydrolysis of phosphatidylinositol-(4,5)-bisphosphate $\left(\mathrm{PIP}_{2}\right)$, which generates inositol$(1,4,5)$-trisphosphate $\left(\mathrm{IP}_{3}\right)$ that initiates a sustained increase in intracellular $\mathrm{Ca}^{2+}$ levels $\left(\left[\mathrm{Ca}^{2+}\right]_{\mathrm{i}}\right)$ that is critical for cytokine production. One mediator of $\mathrm{Ca}^{2+}$ signals in $\mathrm{T}$ cells are the $\mathrm{Ca}^{2+} /$ calmodulin-dependent protein kinase kinases (CaMKKs) that phosphorylate and activate CaM-kinase IV and hence regulate cytokine genes and link $\mathrm{Ca}^{2+}$ signals to microtubule dynamics (Matthews and Cantrell 2009). The CaMKKs also phosphorylate and hence activate the AMP-activated protein kinase (AMPK), a protein kinase with a crucial role in maintaining cellular energy homeostasis (Tamás et al. 2006).
The hydrolysis of $\mathrm{PIP}_{2}$ by phospholipase $\mathrm{C}$ also produces diacyglycerol (DAG) that drives the activation of serine/threonine kinases of the protein kinase $\mathrm{C}(\mathrm{PKC})$ and protein kinase D (PKD) families (Spitaler and Cantrell 2004; Spitaler et al. 2006) (Fig. 1). DAG binding to these kinases is required for catalytic activitation but also mediates the redistribution of PKC and PKD to the membrane. However, the key molecular switch for PKD activation is the PKC-mediated phosphorylation of core serine residues within the PKD catalytic domain. In T lymphocytes, DAG binding proteins also include the Ras guanyl releasing protein (GRP) family of nucleotide exchange factors, which activate Ras GTPases (Stone 2006). In quiescent T lymphocytes, Ras GTPases exist predominantly in an inactive GDP bound state but switch rapidly to an active GTP bound form following engagement of the TCR and regulation of intracellular diacylglycerol levels (Downward

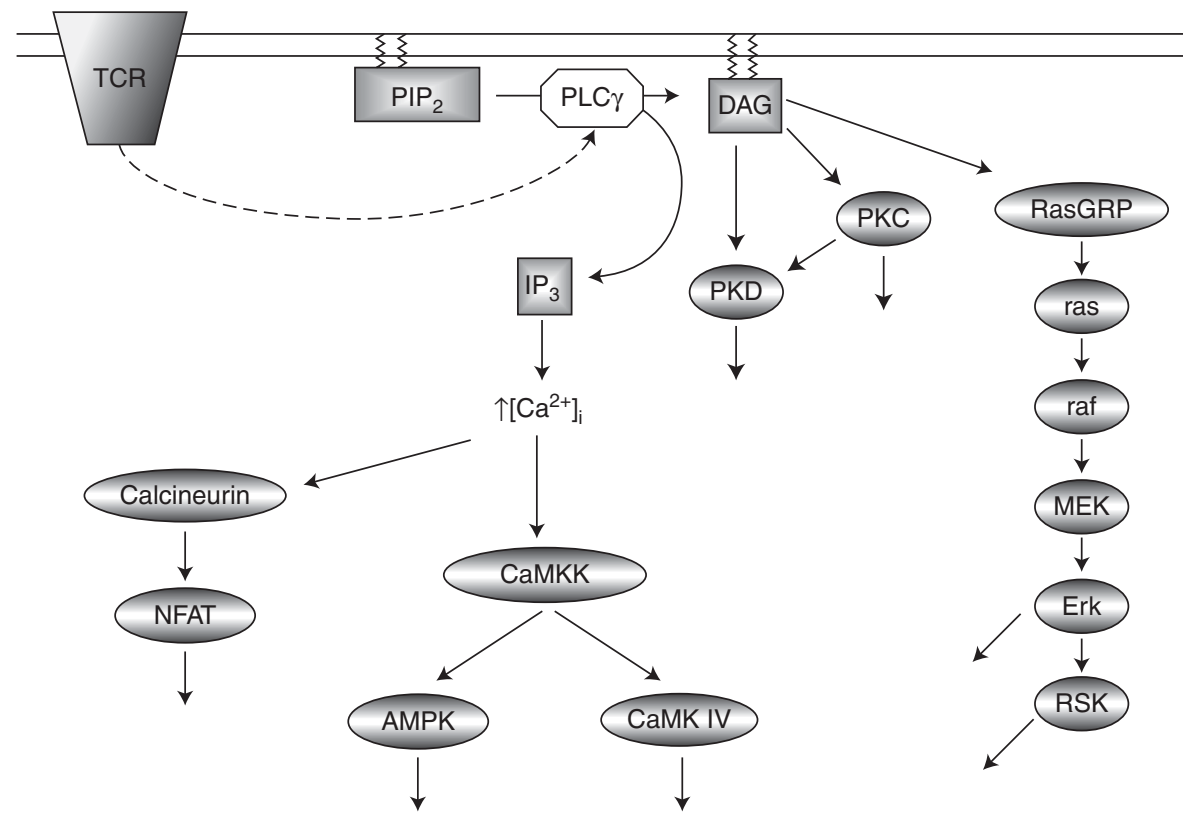

Figure 1. TCR activation of $\mathrm{Ca}^{2+} / \mathrm{DAG}$ regulated kinases. T-cell receptor (TCR) ligation activates phopholipase $\mathrm{C} \gamma(\mathrm{PLC} \gamma)$, which cleaves phosphotidylinositol- $(3,4)$-bisphosphate $\left(\mathrm{PIP}_{2}\right)$ generating two key second messenger molecules, diacylglycerol (DAG) and inositol-(1,4,5)-trisphosphate $\left(\mathrm{IP}_{3}\right) . \mathrm{IP}_{3}$ releases intracellular $\mathrm{Ca}^{2+}$ stores into the cytosol activating; the $\mathrm{Ca}^{2+}$ dependent phosphatase calcineurin inducing the expression of NFAT target genes, and the $\mathrm{Ca}^{2+}$ dependent kinase CaMKK which phosphorylates and activates AMPK and CaMK IV. The accumulation of DAG in the plasma membrane mediates the activation PKC and PKD protein kinases. DAG is also required for the activation of the Erk1/2 and RSK through activation of the Ras GTPase. 
et al. 1990). In their active GTP bound state Ras proteins bind to the serine threonine kinase Raf- 1 and activate the mitogen activated kinase MEK1 that phosphorylates and activates the MAP kinases Erk1 and Erk2 (Matthews and Cantrell 2009). The importance of the Erks for $\mathrm{T}$ cells is well documented and they are a classic example of kinases whose activity is switched on by protein phosphorylation and whose activity is terminated by dephosphorylation.

One other well-characterized serine/threonine kinase whose activity is tightly regulated by phosphorylation during the immune activation of lymphocytes is protein kinase $\mathrm{B}$ ( $\mathrm{PKB}$, also called Akt) (Astoul et al. 2003; Fabre et al. 2005; Finlay et al. 2009; Waugh et al. 2009). The activation of $\mathrm{PKB}$ requires phosphorylation of threonine 308 within the catalytic domain and serine 473 in the carboxyl terminus of the molecule (Pearce et al. 2010). In this context, $\mathrm{PKB} /$ Akt activity is coupled to triggering of antigen and cytokine receptors by the lipid phosphatidylinositol-(3,4,5)-trisphosphate $\left(\mathrm{PIP}_{3}\right)$ (Sinclair et al. 2008) (Fig. 2). Cellular levels of $\mathrm{PIP}_{3}$ are determined by antigen and cytokine receptors that control the activity of class I phosphoinositide 3-kinases (PI3Ks) that phosphorylate the $3^{\prime}-\mathrm{OH}$ position of the inositol ring of phosphatidylinositol- $(4,5)$-bisphosphate (Costello et al. 2002; Sinclair et al. 2008). Extracellular stimuli can also control $\mathrm{PIP}_{3}$ levels and activate PKB in T cells by regulating lipid phosphatases particularily PTEN (phosphatase and tensin homolog deleted on chromosome 10) (Finlay et al. 2009). $\mathrm{PIP}_{3}$ is an important signaling molecule because it binds to the pleckstrin homology $(\mathrm{PH})$ domain of $\mathrm{PKB} /$ Akt (Fig. 2). The rate limiting step for $\mathrm{PKB}$ activation is the phosphorylation of Thr308 within the PKB catalytic domain by 3 phosphoinositide-dependent protein kinase-1 (PDK1) (Pearce et al. 2010). The phosphorylation of serine 473 in the carboxyl terminus of

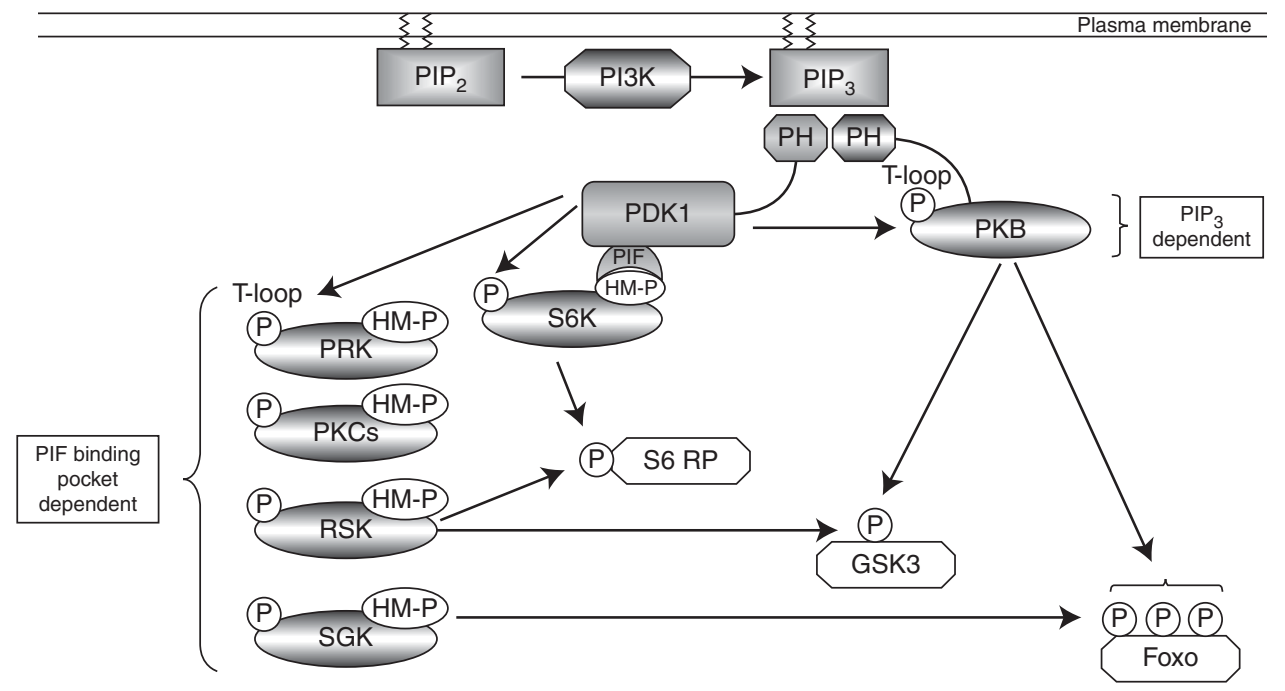

Figure 2. PDK1 signaling. PDK1 acts as a "master kinase" activating multiple AGC kinases through phosphorylation of a conserved Threonine residue ("T-loop" site) within the catalytic domain. PDK1 activation of PKB requires the second messenger molecule $\mathrm{PIP}_{3}$ that accumulates in the plasma membrane because of the action of the lipid kinase PI3K. PDK1 activation of other AGC kinases relies on prior phosphorylation of a Ser/Thr residue within a hydrophobic motif (HM). This phosphorylated motif creates a docking site for PDK1 to bind through its PIF binding pocket, thus allowing PDK1-mediated phosphorylation of the T-loop site and kinase activation. Significant redundancy exists in PDK1 signaling as many downstream substrates, such as S6 ribosomal protein, GSK3, and FoxO transcription factors, can be phosphorylated by multiple PDK1-regulated AGC kinases. 
PKB by the mammalian target of rapamycin (mTOR) complex-2 is also required for optimal kinase activity (Yang et al. 2002; Sarbassov et al. 2005). The link between $\mathrm{PIP}_{3}$ and $\mathrm{PKB}$ activation is that phosphorylation of PKB Thr308 is triggered by increases in cellular $\mathrm{PIP}_{3}$ levels that allow $\mathrm{PIP}_{3}$ binding to the $\mathrm{PH}$ domain of PKB. This is essential to allow PDK1 to phosphorylate Thr308 (Calleja et al. 2007). PDK1 also has a $\mathrm{PH}$ domain that binds $\mathrm{PIP}_{3}$ with high-affinity but it is important to note that $\mathrm{PIP}_{3}$ binding to the PDK1 PH domain is not essential for PDK1 catalytic activity (Bayascas et al. 2008) or the activation of PKB in T cells. However, PDK1 binding to $\mathrm{PIP}_{3}$ at the plasma membrane is required for efficient induction of high levels of $\mathrm{PKB}$ activity, probably by ensuring colocalization of the enzymes (Bayascas et al. 2008; Waugh et al. 2009).

\section{CONSTITUTIVELY ACTIVE SERINE/ THREONINE KINASES IN "QUIESCENT" T-CELLS}

The discussions earlier illustrate the examples of kinases that function as on/off switches in $\mathrm{T}$ cells but there are equally important examples of serine/threonine kinases that have high levels of basal activity in resting $\mathrm{T}$ cells. For example, the rate limiting step in the triggering of $\mathrm{PKB}$ activity is not the activation of PDK1, because this kinase has vey high basal activity, but rather conformational/spatial restraints on the PDK1/Akt interaction (Pearce et al. 2010). In this context, the role of PDK1 extends beyond its ability to link $\mathrm{PIP}_{3}$ to $\mathrm{PKB}$ and includes its ability to phosphorylate and activate p70 ribosomal S6 kinases (S6Ks), p90 ribosomal S6 kinase (RSK), serum and glucocorticoid regulated protein kinases (SGKs), protein kinase Cs, and the protein kinase C-related protein kinases (PRKs) (Williams et al. 2000; Pearce et al. 2010) (Fig. 2). The phosphorylation of S6K1, SGKs, and RSK by PDK1 is regulated by extrinsic stimuli because a key step in the activation of these kinases is their phosphorylation on hydrophobic motifs that create docking sites for the PIF binding pocket in PDK1. For S6K1 the hydrophobic motif kinase is the mTORC1 complex that phosphorylates S6K1 on Thr389. PDK1 can then dock with S6K1 and phosphorylate the kinase at its T-loop site (T229) within the catalytic domain. For SGK1, the hydrophobic motif kinase is the mTOR complex 2 (mTORC2) (Garcia-Martinez and Alessi 2008) whereas RSK activation by PDK1 is controlled by the MAP kinases Erk1/2 (Pearce et al. 2010). The initiating step for RSK activation is thus Erk1/2-mediated phosphorylation of Ser369, Thr365, and Thr577 in the carboxyterminal catalytic domain of the kinase. The activated carboxy-terminal catalytic domain of RSK then phosphorylates Ser386 in RSK to create a docking site for PDK1, which then phosphorylates Serine 227 in the amino-terminal RSK kinase domain thereby activating the enzyme. PDK1 is thus a constitutively active kinase that integrates multiple signaling pathways initiated by antigen receptors and cytokine receptors to control the activity of other AGC kinases (Pearce et al. 2010).

Is the example of $\mathrm{PDK} 1$ constitutive activity unique? No there are other very important instances of constitutively active kinases in $\mathrm{T}$ cells and in some cases these kinases act to restrain T-cell activation. For example, members of the NFAT (nuclear factor of activated T cells) family of transcription factors (NFAT14) are key regulators of cytokine gene expression in T cells. NFATs are phosphorylated in quiescent cells and, as a consequence, are excluded from the nucleus and maintained in an inactive state in the cytosol. NFAT kinases are all constitutively active and include the DYRKs (dualspecificity tyrosine-phosphorylation regulated kinases), casein kinase 1 (CK1), and glycogen synthase kinase 3 (GSK3). The high basal activity of these NFAT kinases in quiescent $\mathrm{T}$ cells keeps NFATs phosphorylated and inactive until triggering of the T-cell antigen receptor raises intracellular free calcium levels and activates the calmodulin-dependent phosphatase calcineurin that then dephosphorylates the NFATs. The dephosphorylation of NFAT allows their translocation to the nucleus and promotes their binding to target genes. The rate limiting step for NFAT activation is thus the activation of a phosphatase: the kinases act as negative 
regulators of NFAT induced gene transcription (Oh-hora and Rao 2008).

\section{REDUNDANCY BETWEEN SERINE/ THREONINE KINASES IN T CELLS}

Studies of the function of serine/threonine kinases in $\mathrm{T}$ cells have been complicated by the fact that many cells coexpress multiple isoforms of a kinase making it difficult to assess the function of these molecules with simple single-gene deletion strategies. For example, there are three isoforms of PKB: These may have unique roles but are also quite redundant with each other. Hence, in T-cell progenitors in the thymus there is very little impact of deleting a single $\mathrm{PKB}$ isoform; the deletion of two isoforms $\mathrm{PKB} \alpha$ and PKB $\beta$ inhibits cell proliferation whereas the combined deletion of all three $\mathrm{PKB}$ isoforms inhibits the T-cell survival response (Fayard et al. 2007; Juntilla et al. 2007; Mao et al. 2007). There can even be redundancy among different members of a family of kinases. For example, the p90 ribosomal S6 kinase (RSK) can substitute for p70 ribosomal S6 kinases (S6Ks) and converge to phosphorylate the S6 ribosomal subunit, a key component of the translational machinery in eukaryotic cells that is essential for ribosome biogenesis (Salmond et al. 2009). Additionally, both PKB and SGK can phosphorylate and inactivate FoxO transcription factors (Brunet et al. 2001; Burgering 2008) (Fig. 2).

One way to evade issues of kinase redundancy and gain an understanding of the functional importance of a kinase family is to delete a rate limiting upstream regulator that will simultaneously inactivate multiple members of a kinase family. In this context, PDK1 phosphorylates and activates multiple members of the AGC kinase family including PKB/Akt, RSK, S6K, and PRKs. The deletion of PDK1 can thus eliminate the activity of multiple AGC family kinases (Lawlor et al. 2002). Accordingly, studies that explored the impact of PDK1 deletion in T-cell progenitors in the thymus have been much more informative about the role of AGC kinases in T cells than studies of $\mathrm{T}$ cell lacking individual members of this kinase family (Hinton et al. 2004). Hence the differentiation and proliferative expansion of pre-T cells in the thymus is dependent on signaling pathways mediated by PDK1. However, the substitution of wild-type PDK1 with a PDK1 L155E mutant that supports full PKB activation in $\mathrm{T}$ cells is not sufficient to restore normal thymus function (Kelly et al. 2007). The substitution of leucine (L) 155 in PDK1 with glutamate (E) disrupts the integrity of an important PDK1 domain termed the PIF binding pocket. This domain is not required for $\mathrm{PKB}$ phosphorylation but is necessary for PDK1 to interact with phosphorylated carboxy-terminal hydrophobic motifs in S6Ks and RSK and is necessary for PDK1 to activate these AGC kinases by phosphorylating their catalytic domains (Collins et al. 2003). A comparison of the function of wild type and the PDK1 L155E mutant thus reveals how multiple AGC serine kinases and not just $\mathrm{PKB}$ are important in T-cells (Kelly et al. 2007).

The ability of PDK1 to control the activity of many AGC kinases has generated the concept that some kinases act as "master" kinases. It is not yet clear how general this concept is for $\mathrm{T}$ cells but there is at least one more example that should be considered. Hence, LKB1 (or serine/threonine kinase 11-STK11) is a kinase that phosphorylates critical activating residues in the catalytic domains of multiple members of the AMPK family including the $\alpha 1$ and $\alpha 2$ isoforms of AMPK and NUAK1-2, BRSK1-2, QIK, QSK, SIK, MELK, and MARK1-4 kinases (Alessi et al. 2006) (Fig. 3). These kinases are thought to be essential to control cell polarity and to control cellular responses to energy stress. However, elimination of single members of the AMPK family of kinases such as deletion of AMPK $\alpha 1$ or loss of single MARK kinases has only had a relatively minor effect on peripheral $\mathrm{T}$ cells and causes no problems for T-cell development in the thymus (Mayer et al. 2008; Lin et al. 2009). This could indicate that AMPK family kinases are physiologically irrelevant for T-cell biology but the data are equally consistent with the idea that there is redundancy among different AMPK family members for both energy metabolism and the control of T-cell polarity. In this respect, T-lineage specific 
D. Finlay and D. Cantrell

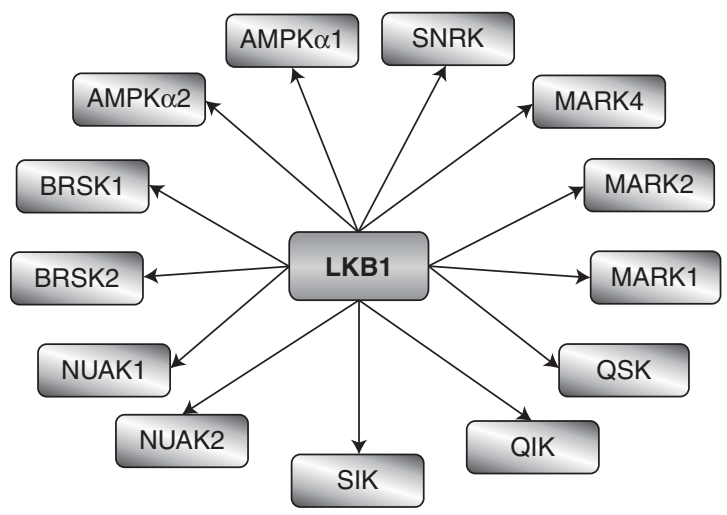

Figure 3. LKB1 is a "master kinase.” LKB1 phosphorylates and activates 13 kinases within the AMPK family of protein kinases.

deletion of LKB1 shows that LKB1 is essential for the survival and development of TCR $\beta$ selected T-cell progenitors in the thymus and is also required for survival and proliferation of peripheral CD8 T cells (Cao et al.; Tamás et al. 2010). Importantly, LKB1 appears to be required for several key metabolic processes in $\mathrm{T}$ cells. For example, LKB1 controls expression of CD98, a key subunit of the L-system amino acid transporter in $\mathrm{T}$ cells and is also required to sustain the phosphorylation of the ribosomal S6 subunit (Tamás et al. 2010). As discussed, the role of LKB1 is to phosphorylate and activate multiple AMPK family members. To date there are no reports that the loss of a single AMPK isoform phenocopies the loss of LKB1 (Mayer et al. 2008). Hence it appears that there is in vivo redundancy among different AMPK family members for the control of T-cell metabolism; the elimination of LKB1 circumvents this redundancy by simultaneously eliminating activation of multiple AMPK family members (Tamás et al. 2010).

\section{PROTEIN PHOSPHORYLATION INT CELLS: A LINK BETWEEN THE PLASMA MEMBRANE AND THE NUCLEUS}

One of the first examples of how a pathway of protein phosphorylation could couple signals generated at the plasma membrane to events in the cell nucleus came from studies of NFATs that are cytosolic and inactive when phosphorylated and only localized in the nucleus and able to regulate their target genes when they are dephosphorylated (Hogan et al. 2003) (Fig. 4). NFAT dephosphorylation is regulated by the TCR that controls intracellular calcium levels and the activity of the calcium phosphatatase calcineurin (Oh-hora and Rao 2008). In the case of NFAT protein, phosphorylation is a negative regulator of transcriptional activity. However, there are also examples of pathways of protein phosphorylation that positively regulate gene transcription notably from studies of the MAP kinases. Hence, Erk1/2 phosphorylate and activate the ternary complex factor (TCF) subfamily of ETS-domain transcription factors Elk-1, SAP1, and SAP2 that control immediate early gene expression in $\mathrm{T}$ cells (e.g., c-fos, Egr1, and EgR3) (Costello et al. 2004). The TCFs also control the transcription of dual specificity (Thr/Tyr) MAPK phosphatases that initiate a negative regulatory feedback pathway that acts to limit the duration and magnitude of ERK activity (Owens and Keyse 2007).

The consideration of NFAT regulation illustrates that one key way in which protein phosphorylation is important to control cell function is that it can promote protein interactions that can modify protein activity and subcellular localization (Fig. 4). In this context, 14-3-3 proteins (14-3-3s) are highly conserved dimeric molecules that bind to phosphorylated 


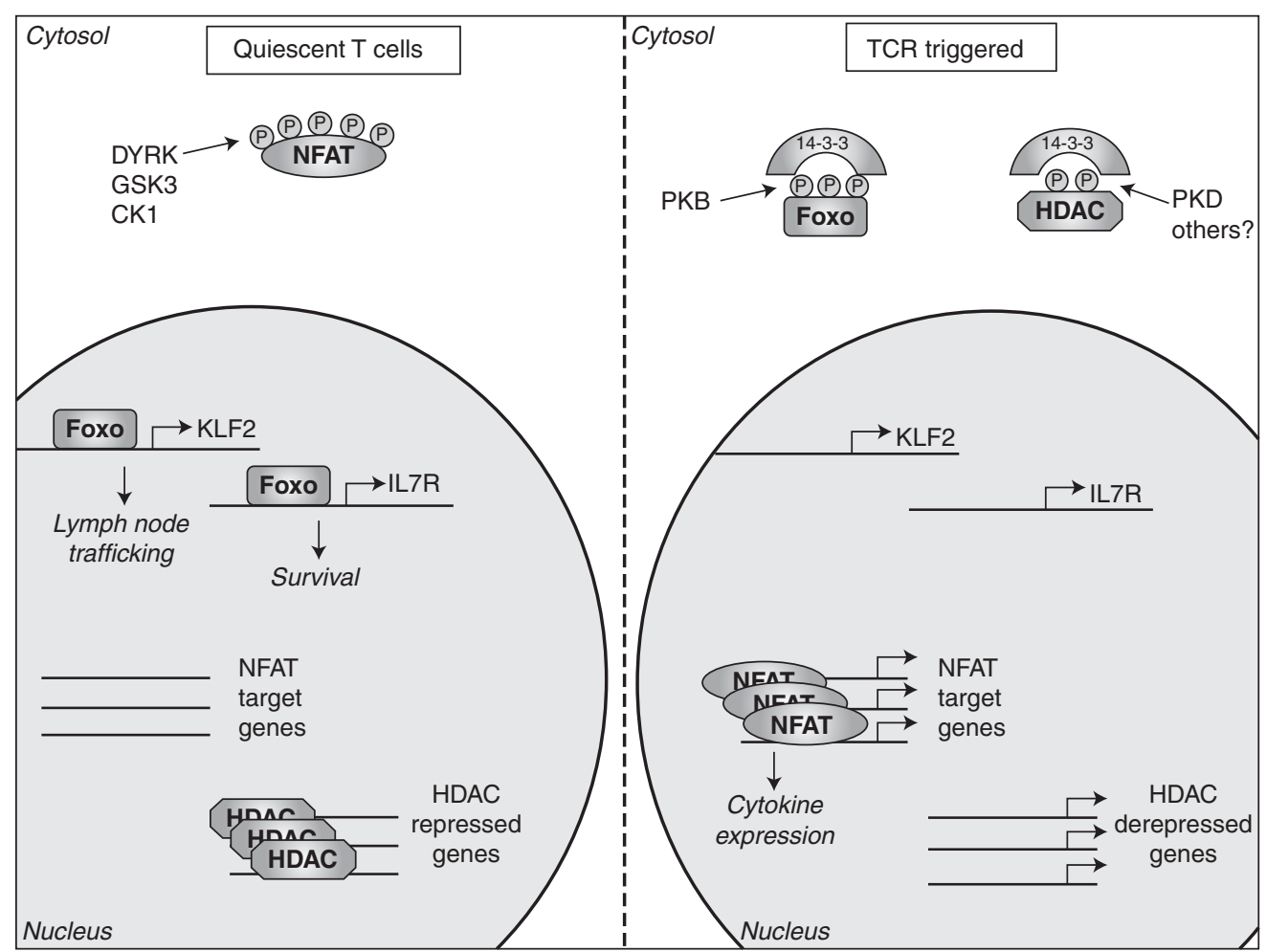

Figure 4. Phosphorylation determined intracellular localization. In quiescent $\mathrm{T}$ cells constitutively active kinases (DYRK, GSK3, and CK1) maintain NFAT transcription factors in a phosphorylated state excluding them from the nucleus. In contrast, the kinases that regulate FoxO transcription factors and histone deacetylases (HDACs) are inactive in quiescent cells allowing FoxOs to access the nucleus and control genes involved in T-cell trafficking and survival whereas HDACs repress gene expression through modulating chromatin structure. Following triggering of the T-cell receptor (TCR) FoxO transcription factors and HDACs are phosphorylated, resulting in their cytosolic sequestration by 14-3-3 proteins, thus preventing their nuclear actions. Meanwhile, the $\mathrm{Ca}^{2+}$ activated phosphatase, calcineurin, dephosphorylates NFAT transcription factors allowing nuclear entry and the expression of key cytokine genes.

serine and threonine sites in a wide range of proteins (Johnson et al.; Dubois et al. 2009; Morrison 2009). 14-3-3s target proteins include transcription factors, chromatin regulators, enzymes, and structural components of vesicle and protein trafficking. In lymphocytes, one example of how protein phosphorylation and 14-3-3 proteins can coordinate programs of gene expression is in the context of class II histone deacetylases (HDACs) (Martin et al. 2007). These control the acetylation/deacetylation of nucleosomal histones, regulate chromatin accessibility and also control gene activity by repressing the activity of MEF2 transcription factors. The suppressive effects of class II
HDACs on gene transcription can be relieved by triggering of the antigen receptor that leads to the phosphorylation of serine residues within the amino-termini of the HDACs (Matthews et al. 2006; Martin et al. 2007). The proposed HDAC kinase is the diacyglycerol-regulated kinase protein kinase D. Phosphorylation at the regulatory sites promotes the redistribution of class II HDACs from the nucleus to the cytosol allowing them to bind to 14-3-3 complexes thus disrupting repressive interactions between HDACs and histones and/or transcription factors and allowing gene transcription to occur (Matthews et al. 2006). The regulated protein phosphorylation of class II HDACs is thus a 
key epigenetic control mechanism that determines gene expression in lymphocytes and HDAC-regulated genes in $\mathrm{T}$ cells are extensive and include genes that control both positive and negative selection of thymocytes (Kasler and Verdin 2007) (Fig. 4).

Another evolutionarily conserved set of transcription factors controlled by protein phosphorylation and 14-3-3s in lymphocytes are the Forkhead box $\mathrm{O}$ (FoxO) family of transcription factors FoxO 1, 3a, and 4 (Burgering 2008). Nonphosphorylated FoxO 1, 3a, and 4 are found in the nuclei of quiescent $\mathrm{T}$ cells where they drive transcription of genes encoding cytokine receptor and transcription factors (Coffer and Burgering 2004; Fabre et al. 2008; Kerdiles et al. 2009). For example, FoxOs drive expression of the receptor for interleukin 7, an essential homeostatic cytokine for $\mathrm{T}$ cells (Kerdiles et al. 2009; Ouyang et al. 2009). FoxO activity in naïve and memory $\mathrm{T}$ cells also controls the expression of the transcription factor KLF2 that directly regulates the gene transcription of key adhesion molecules and chemokine receptors that control T-cell entry and egress from secondary lymphoid tissues (Carlson et al. 2006; Finlay et al. 2009; Kerdiles et al. 2009; Waugh et al. 2009) (Fig. 4). FoxO 1 and 3a are substrates for PKB and their phosphorylation results in their nuclear export; phosphorylated FoxO transcription factors are then retained in the cytosol in a complex with 14-3-3 proteins ensuring the termination of FoxO-mediated gene transcription (Carlson et al. 2006; Burgering 2008) (Fig. 4).

The importance of PKB-mediated phosphorylation and inactivation of FoxOs is easily illustrated. First, the deletion of FoxOs in naïve $\mathrm{T}$ cells disrupts T-cell homing and survival (Kerdiles et al. 2009; Ouyang et al. 2009). It is also clear that much of the T-cell pathology caused by deletion of the tumor suppressor PTEN (phosphatase and tensin homolog deleted on chromosome 10) can be ascribed to FoxO deregulation. PTEN is a lipid phosphatase with specificity for the $3^{\prime}$ position of $\mathrm{PIP}_{3}$ and its deletion causes $\mathrm{T}$ cells to accumulate high levels of $\mathrm{PIP}_{3}$ and hence drives PDK1-mediated phosphorylation and activation of $\mathrm{PKB}$ with the resultant phosphorylation and inactivation of the FoxO1, 3a, and 4 (Finlay et al. 2009). This loss of FoxO transcriptional activity immediately causes loss of KLF2 expression and loss of expression of the KLF2 targets L-selectin/CD62L and CCR7 (Finlay et al. 2009). CD62L/L-selectin plays a fundamental role controlling the transmigration of $\mathrm{T}$ cells across high endothelial venules (Arbones et al. 1994) and the chemokine receptor CCR7 controls lymphocyte entry and retention in lymphoid tissues (Forster et al. 2008). Consequently the loss of FoxO activity in PTEN null cells changes the expression of key trafficking receptors in $\mathrm{T}$ cells and prevents these from cells undergoing their normal migration from blood into secondary lymphoid tissue (Finlay et al. 2009). These trafficking defects are not observed following deletion of PDK1, which causes loss of FoxO phosphorylation and restores FoxO transcriptional activity (Finlay et al. 2009). It is also striking that the activation of PDK1-mediated protein phosphorylation in PTEN null cells is key for T lymphomagenesis. Hence the tissue specific deletion of PTEN in thymocytes using Cre-loxP strategies results in $\mathrm{T}$ leukemogenesis or lymphomagenesis but PTEN null T-cell progenitors cannot transform or develop into invasive and fatal $\mathrm{T}$ lymphoma without PDK1 (Finlay et al. 2009).

\section{CONCLUDING REMARKS}

Recent studies have begun to reveal how protein phosphorylation reversibly regulates key processes in T lymphocytes. However, we have only uncovered a fraction of the relevant information and progress is constantly hampered by technical difficulties. It is still relatively difficult to study protein phosphorylation and the rationale for focusing on particular pathways of protein phosphorylation frequently stems from reagent availability rather than any intellectual deliberation. For example, the MAP kinases Erk1/2 are particularly well studied in T cells for pragmatic reasons: There are high quality phosphospecific monoclonal antibodies that selectively recognize phosphorylated and activated Erks and that can be used for single-cell analysis 
of ERK activity using flow cytometry. It is much harder to study many other kinase pathways using analogous reagents. However, as advances in technology reveal the magnitude and complexity of the T-cell phospho-proteome it is becoming clear that there are unique functions in $\mathrm{T}$ cells for ubiquitously expressed serine/ threonine kinases. It is also clear that studies of serine/threonine kinases can generate a molecular understanding of how signal transduction pathways control T-cell function. Such studies have the added advantage that they provide a clear indication of the molecules that might be useful targets for manipulation of the immune response.

\section{REFERENCES}

Alessi DR, Sakamoto K, Bayascas JR. 2006. LKB1-dependent signaling pathways. Annu Rev Biochem 75: 137-163.

Arbones ML, Ord DC, Ley K, Ratech H, Maynard-Curry C, Otten G, Capon DJ, Tedder TF. 1994. Lymphocyte homing and leukocyte rolling and migration are impaired in L-selectin-deficient mice. Immunity 1: 247-260.

Astoul E, Laurence AD, Totty N, Beer S, Alexander DR, Cantrell DA. 2003. Approaches to define antigen receptor-induced serine kinase signal transduction pathways. J Biol Chem 278: 9267-9275.

Bayascas JR, Wullschleger S, Sakamoto K, Garcia-Martinez JM, Clacher C, Komander D, van Aalten DM, Boini KM, Lang F, Lipina C, et al. 2008. Mutation of the PDK1 PH domain inhibits protein kinase B/Akt, leading to small size and insulin resistance. Mol Cell Biol 28: 3258-3272.

Brunet A, Park J, Tran H, Hu LS, Hemmings BA, Greenberg ME. 2001. Protein kinase SGK mediates survival signals by phosphorylating the forkhead transcription factor FKHRL1 (FOXO3a). Mol Cell Biol 21: 952-965.

Burgering BM. 2008. A brief introduction to FOXOlogy. Oncogene 27: 2258-2262.

Calleja V, Alcor D, Laguerre M, Park J, Vojnovic B, Hemmings BA, Downward J, Parker PJ, Larijani B. 2007. Intramolecular and intermolecular interactions of protein kinase B define its activation in vivo. PLoS Biol 5: e95.

Cao Y, Li H, Liu H, Zheng C, Ji H, Liu X. 2010. The serine/ threonine kinase LKB1 controls thymocyte survival through regulation of AMPK activation and Bcl-XL expression. Cell Res 20: 99-108.

Carlson CM, Endrizzi BT, Wu J, Ding X, Weinreich MA, Walsh ER, Wani MA, Lingrel JB, Hogquist KA, Jameson SC. 2006. Kruppel-like factor 2 regulates thymocyte and T-cell migration. Nature 442: 299-302.

Carrascal M, Ovelleiro D, Casas V, Gay M, Abian J. 2008. Phosphorylation analysis of primary human T-lymphocytes using sequential IMAC and titanium oxide enrichment. Jf Proteome Res 7: 5167-5176.
Coffer PJ, Burgering BM. 2004. Forkhead-box transcription factors and their role in the immune system. Nat Rev Immunol 4: 889-899.

Collins BJ, Deak M, Arthur JS, Armit LJ, Alessi DR. 2003. In vivo role of the PIF-binding docking site of PDK1 defined by knock-in mutation. Embo J 22: 4202-4211.

Costello PS, Gallagher M, Cantrell DA. 2002. Sustained and dynamic inositol lipid metabolism inside and outside the immunological synapse. Nat Immunol 3: 1082-1089.

Costello PS, Nicolas RH, Watanabe Y, Rosewell I, Treisman R. 2004. Ternary complex factor SAP-1 is required for Erk-mediated thymocyte positive selection. Nat Immunol 5: 289-298.

Downward J, Graves JD, Warne PH, Rayter S, Cantrell DA. 1990. Stimulation of p21ras upon T-cell activation. Nature 346: 719-723.

Dubois F, Vandermoere F, Gernez A, Murphy J, Toth R, Chen S, Geraghty KM, Morrice NA, MacKintosh C. 2009. Differential 14-3-3 affinity capture reveals new downstream targets of phosphatidylinositol 3-kinase signaling. Mol Cell Proteomics 8: 2487-2499.

Fabre S, Carrette F, Chen J, Lang V, Semichon M, Denoyelle C, Lazar V, Cagnard N, Dubart-Kupperschmitt A, Mangeney $\mathrm{M}$, et al. 2008. FOXO1 regulates L-Selectin and a network of human T-cell homing molecules downstream of phosphatidylinositol 3-kinase. J Immunol 181: $2980-2989$.

Fabre S, Lang V, Harriague J, Jobart A, Unterman TG, Trautmann A, Bismuth G. 2005. Stable activation of phosphatidylinositol 3-kinase in the T-cell immunological synapse stimulates Akt signaling to FoxO1 nuclear exclusion and cell growth control. J Immunol 174: 4161-4171.

Fayard E, Gill J, Paolino M, Hynx D, Hollander GA, Hemmings BA. 2007. Deletion of PKBalpha/Akt1 Affects Thymic Development. PLoS ONE 2: e992.

Finlay DK, Sinclair LV, Feijoo C, Waugh CM, Hagenbeek TJ, Spits H, Cantrell DA. 2009. Phosphoinositide-dependent kinase 1 controls migration and malignant transformation but not cell growth and proliferation in PTEN-null lymphocytes. J Exp Med 206: 2441-2454.

Forster R, Davalos-Misslitz AC, Rot A. 2008. CCR7 and its ligands: Balancing immunity and tolerance. Nat Rev Immunol 8: 362-371.

Garcia-Martinez JM, Alessi DR. 2008. mTOR complex 2 (mTORC2) controls hydrophobic motif phosphorylation and activation of serum- and glucocorticoidinduced protein kinase 1 (SGK1). Biochem J 416: 375385.

Hinton HJ, Alessi DR, Cantrell DA. 2004. The serine kinase phosphoinositide-dependent kinase 1 (PDK1) regulates T-cell development. Nat Immunol 5: 539-545.

Hogan PG, Chen L, Nardone J, Rao A. 2003. Transcriptional regulation by calcium, calcineurin, and NFAT. Genes Dev 17: 2205-2232.

Johnson C, Crowther S, Stafford MJ, Campbell DG, Toth R, MacKintosh C. Bioinformatic and experimental survey of 14-3-3-binding sites. Biochem J 427: 69-78.

Juntilla MM, Wofford JA, Birnbaum MJ, Rathmell JC, Koretzky GA. 2007. Akt1 and Akt2 are required for $\alpha \beta$ thymocyte survival and differentiation. Proc Natl Acad Sci 104: 12105-12110. 
D. Finlay and D. Cantrell

Kasler HG, Verdin E. 2007. Histone deacetylase 7 functions as a key regulator of genes involved in both positive and negative selection of thymocytes. Mol Cell Biol 27: 5184-5200.

Kelly AP, Finlay DK, Hinton HJ, Clarke RG, Fiorini E, Radtke F, Cantrell DA. 2007. Notch-induced T-cell development requires phosphoinositide-dependent kinase 1. Embo J 26: 3441-3450.

Kerdiles YM, Beisner DR, Tinoco R, Dejean AS, Castrillon DH, DePinho RA, Hedrick SM. 2009. FoxO1 links homing and survival of naive T-cells by regulating L-selectin, CCR7 and interleukin 7 receptor. Nat Immunol 10: 176- 184 .

Lawlor MA, Mora A, Ashby PR, Williams MR, Murray-Tait V, Malone L, Prescott AR, Lucocq JM, Alessi DR. 2002. Essential role of PDK1 in regulating cell size and development in mice. Embo J 21: 3728-3738.

Lin J, Hou KK, Piwnica-Worms H, Shaw AS. 2009. The polarity protein Par1b/EMK/MARK2 regulates T-cell receptor-induced microtubule-organizing center polarization. J Immunol 183: 1215-1221.

Mao C, Tili EG, Dose M, Haks MC, Bear SE, Maroulakou I, Horie K, Gaitanaris GA, Fidanza V, Ludwig T, et al. 2007. Unequal contribution of Akt isoforms in the doublenegative to double-positive thymocyte transition. $J$ Immunol 178: 5443-5453.

Martin M, Kettmann R, Dequiedt F. 2007. Class IIa histone deacetylases: regulating the regulators. Oncogene 26: 5450-5467.

Matthews SA, Cantrell DA. 2009. New insights into the regulation and function of serine/threonine kinases in $\mathrm{T}$ lymphocytes. Immunol Rev 228: 241-252.

Matthews SA, Liu P, Spitaler M, Olson EN, McKinsey TA, Cantrell DA, Scharenberg AM. 2006. Essential role for protein kinase $\mathrm{D}$ family kinases in the regulation of class II histone deacetylases in B lymphocytes. Mol Cell Biol 26: 1569-1577.

Mayer A, Denanglaire S, Viollet B, Leo O, Andris F. 2008 AMP-activated protein kinase regulates lymphocyte responses to metabolic stress but is largely dispensable for immune cell development and function. Eur J Immunol 38: 948-956.

Mayya V, Lundgren DH, Hwang SI, Rezaul K, Wu L, Eng JK, Rodionov V, Han DK. 2009. Quantitative phosphoproteomic analysis of $\mathrm{T}$-cell receptor signaling reveals system-wide modulation of protein-protein interactions. Sci Signaling 2: ra46.

Morrison DK. 2009. The $14-3-3$ proteins: integrators of diverse signaling cues that impact cell fate and cancer development. Trends Cell Biol 19: 16-23.

Nguyen V, Cao L, Lin JT, Hung N, Ritz A, Yu K, Jianu R, Ulin SP, Raphael BJ, Laidlaw DH, et al. 2009. A new approach for quantitative phosphoproteomic dissection of signaling pathways applied to T-cell receptor activation. Mol Cell Proteomics 8: 2418-2431.
Oh-hora M, Rao A. 2008. Calcium signaling in lymphocytes. Current Opin Immunol 20: 250-258.

Ouyang W, Beckett O, Flavell RA, Li MO. 2009. An essential role of the Forkhead-box transcription factor FoxO1 in control of T-cell homeostasis and tolerance. Immunity 30: $358-371$.

Owens DM, Keyse SM. 2007. Differential regulation of MAP kinase signalling by dual-specificity protein phosphatases. Oncogene 26: 3203-3213.

Pearce LR, Komander D, Alessi DR. 2010. The nuts and bolts of AGC protein kinases. Nat Rev Mol Cell Biol 11: 9-22.

Salmond RJ, Emery J, Okkenhaug K, Zamoyska R. 2009. MAPK, phosphatidylinositol 3-kinase, and mammalian target of rapamycin pathways converge at the level of ribosomal protein S6 phosphorylation to control metabolic signaling in CD8 T-cells. J Immunol 183: $7388-7397$.

Sarbassov DD, Guertin DA, Ali SM, Sabatini DM. 2005. Phosphorylation and regulation of $\mathrm{Akt} / \mathrm{PKB}$ by the rictor-mTOR complex. Science 307: 1098-1101.

Sinclair LV, Finlay D, Feijoo C, Cornish GH, Gray A, Ager A, Okkenhaug K, Hagenbeek TJ, Spits H, Cantrell DA. 2008. Phosphatidylinositol-3-OH kinase and nutrient-sensing mTOR pathways control T-lymphocyte trafficking. Nat Immunol 9: 513-521.

Spitaler M, Cantrell DA. 2004. Protein kinase C and beyond. Nat Immunol 5: 785-790.

Spitaler M, Emslie E, Wood CD, Cantrell D. 2006. Diacylglycerol and protein kinase D localization during T-lymphocyte activation. Immunity 24: 535-546.

Stone JC. 2006. Regulation of Ras in lymphocytes: get a GRP. Biochem Soc Trans 34: 858-861.

Tamás P, Hawley SA, Clarke RG, Mustard KJ, Green K, Hardie D, Cantrell DA. 2006. Regulation of the energy sensor AMP-activated protein kinase by antigen receptor and $\mathrm{Ca} 2+$ in T-lymphocytes. J Exp Med 203: 1665-1670.

Tamás P, Macintyre A, Finlay D, Clarke R, Feijoo-Carnero C, Ashworth A, Cantrell D. 2010. LKB1 is essential for the proliferation of T-cell progenitors and mature peripheral T-cells. Eur J Immunol 40: 242-253.

Waugh C, Sinclair L, Finlay D, Bayascas J, Cantrell D. 2009. PIP3 binding to Phosphoinositide dependent kinase 1 regulates a protein kinase $\mathrm{B} / \mathrm{Akt}$ signalling threshold that dictates T-cell migration not proliferation. Mol Cell Biol 29: 5952-5962.

Williams MR, Arthur JS, Balendran A, van der Kaay J, Poli V, Cohen P, Alessi DR. 2000. The role of 3-phosphoinositide-dependent protein kinase 1 in activating AGC kinases defined in embryonic stem cells. Curr Biol 10: 439-448.

Yang J, Cron P, Thompson V, Good V, Hess D, Hemmings B, Barford D. 2002. Molecular mechanism for the regulation of protein kinase B/Akt by hydrophobic motif phosphorylation. Mol Cell 9: 1227-1240. 


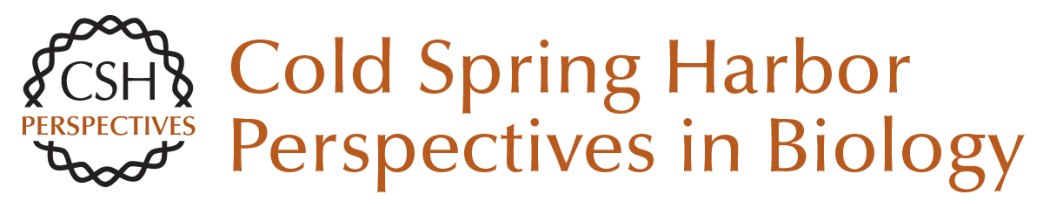

\section{The Coordination of T-cell Function by Serine/Threonine Kinases}

David Finlay and Doreen Cantrell

Cold Spring Harb Perspect Biol 2011; doi: 10.1101/cshperspect-a002261 originally published online October 20, 2010

\section{Subject Collection Immunoreceptor Signaling}

The Coordination of T-cell Function by Serine/Threonine Kinases

David Finlay and Doreen Cantrell

ITAM-mediated Signaling by the T-Cell Antigen Receptor

Paul E. Love and Sandra M. Hayes

Coordination of Receptor Signaling in Multiple Hematopoietic Cell Lineages by the Adaptor

Protein SLP-76

Martha S. Jordan and Gary A. Koretzky

The Cytoskeleton Coordinates the Early Events of B-cell Activation

Naomi E. Harwood and Facundo D. Batista

An Enigmatic Tail of CD28 Signaling

Jonathan S. Boomer and Jonathan M. Green

\section{Mediation of T-Cell Activation by Actin}

Meshworks

Peter Beemiller and Matthew F. Krummel

T-Cell Signaling Regulated by the Tec Family

Kinase, Itk

Amy H. Andreotti, Pamela L. Schwartzberg, Raji E. Joseph, et al.
Perspectives for Computer Modeling in the Study of T Cell Activation Jesse Coward, Ronald N. Germain and Grégoire Altan-Bonnet

Structural Biology of the T-cell Receptor: Insights into Receptor Assembly, Ligand Recognition, and Initiation of Signaling

Kai W. Wucherpfennig, Etienne Gagnon, Melissa J. Call, et al.

Src-family and Syk Kinases in Activating and

Inhibitory Pathways in Innate Immune Cells:

Signaling Cross Talk Clifford A. Lowell

The LAT Story: A Tale of Cooperativity, Coordination, and Choreography

Lakshmi Balagopalan, Nathan P. Coussens, Eilon Sherman, et al.

Antigen Receptor Signaling to NF- $\mathrm{kB}$ via CARMA1, BCL10, and MALT1 Margot Thome, Jean Enno Charton, Christiane Pelzer, et al.

It's All About Change: The Antigen-driven Initiation of B-Cell Receptor Signaling Wanli Liu, Hae Won Sohn, Pavel Tolar, et al.

ZAP-70: An Essential Kinase in T-cell Signaling Haopeng Wang, Theresa A. Kadlecek, Byron B. Au-Yeung, et al.

For additional articles in this collection, see http://cshperspectives.cshlp.org/cgi/collection/

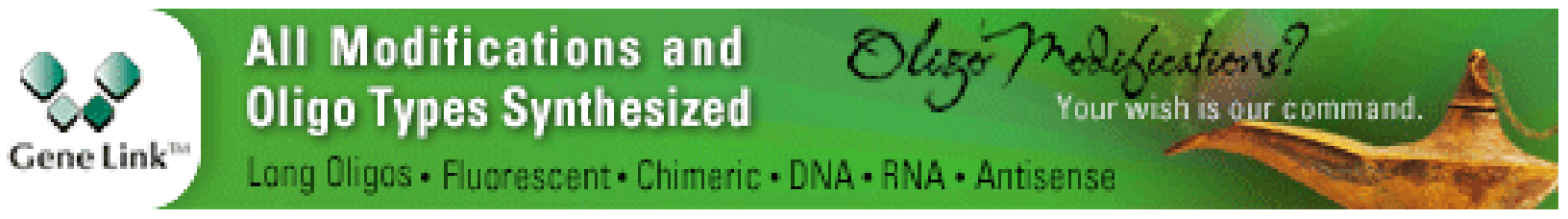




\section{Lipid Signaling in T-Cell Development and Function \\ Yina H. Huang and Karsten Sauer}

Understanding the Structure and Function of the Immunological Synapse

Michael L. Dustin, Arup K. Chakraborty and Andrey S. Shaw

For additional articles in this collection, see http://cshperspectives.cshlp.org/cgi/collection/

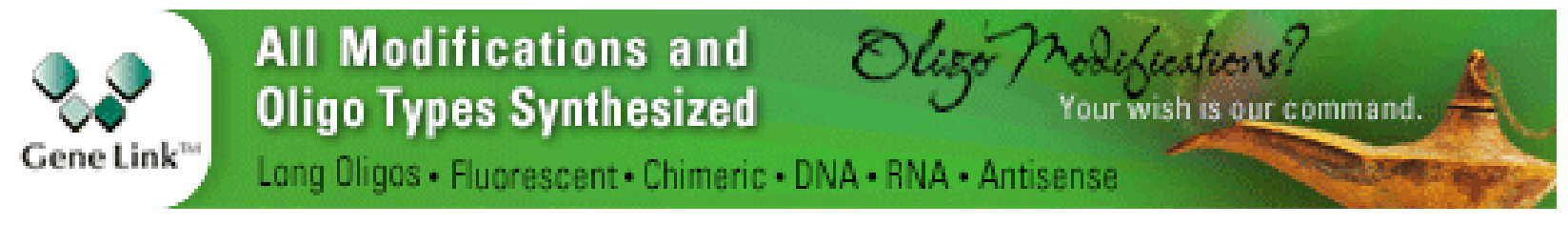

Copyright @ 2011 Cold Spring Harbor Laboratory Press; all rights reserved 